\title{
COMPARAISON DES PERFORMANCES EN PISCICULTURE D'UN HYBRIDE INTRA-SPÉCIFIQUE
(6) SAUVAGE $\times$ O DOMESTIQUE) ET DE LA SOUCHE DOMESTIQUE CHEZ LA TRUITE COMMUNE (SALMO TRUTTA). ESSAI PRÉLIMINAIRE D'IMPLANTATION EN RUISSEAU.
}

\author{
G. MAISSE (1), J.P. PORCHER (2), A. NIHOUARN (2), B. CHEVASSUS (3)
} Avec la collaboration technique de V. FOLMER (4).

\section{RÉSUME :}

Les performances en élevage puis en ruisseau d'un hybride intra-spécifique ( $O$ sauvage $\times$ Qdomestique) de Truite commune (Salmo trutta) ont été comparées à celles de la souche maternelle mise dans des conditions identiques.

Il est apparu

1) que dans la phase d'élevage, l'hybride présente une moins bonne croissance et une plus grande sensibilité à la costiose que la souche maternelle, à relier à des performances moindres lors de la résorption vésiculaire et du début de l'alimentation.

2) que dans la phase en ruisseau, le taux d'implantation de chaque origine dépend des caractéristiques du milieu récepteur.

L'utilisation d'une telle hybridation en vue des empoissonnements des eaux libres est discutée en fonction de l'objectif visé.

\section{SUMMARY :}

Performances, in hatchery and then in streams, of an interstrain hybrid (wild $\sigma_{x}$ domestic $Q$ ) of brown trout ( $S a l m o$ trutta) are compared with those of the maternal strain in the same conditions.

It is shown :

1) that during rearing period the hybrid grows less than the maternal strain and is more sensitive to costia in relation with less performances during yolk sac resorption and start feeding.

2) that during stream period implantation level for each origin depends on the habitat.

The use of such a hybridisation for stocking wild waters is discussed in terms of several planting strategies

\section{INTRODUCTION :}

Dès 1873, STONE, cité par VINCENT (1960) soupçonnait que la domestication des Truites pouvait modifier leur comportement dans le milieu naturel et notamment dans leur recherche de la nourriture. Un certain nombre d'auteurs dont GREENE (1952). VINCENT (1960) et FLICK et WEBSTER (1964 et 1976), ont étudié les performances comparées de souches, domestiques d'une part et sauvages d'autre part, d'Omble de fontaine, Salvelinus fontinalis, déversées en ruisseau ou en lac.

Dans la plupart de ces cas les animaux d'origine sauvage s'implantent mieux que les souches domestiques, l'importance relative des deux origines étant cependant étroitement dépendante des caractéristiques du milieu récepteur, et notamment, en ruisseau, de la vitesse du courant.

(1) I.N.R.A., Laboratoire de Physiologie des Poissons - 35042 RENNES CEDEX.

(2) Conseil Supérieur de la Pêche. Délégation Régionale no $2-84$, rue de Rennes - 35510 CESSON SÉVIGNE.

(3) I.N.R.A., Laboratoire de Physiologie des Poissons - 78350 JOUY-EN-JOSAS

(4) Fédération des A.A.P.P. d'llle-et-Vilaine. 
Ces différences sont à rapprocher des résultats des tests de rusticité (vitesse de nage) obtenus par GREENE (1964) qui sont en faveur des souches sauvages. Toutes ces observations vont dans le sens de la réflexion de GREENE qui en conclusion de son article de 1952 écrivait : (traduction des auteurs) "Eu égard à l'existence, reconnue de tous, de nombreuses souches distinctes de Truite à la fois dans les eaux naturelles et dans les piscicultures, il paraît surprenant et paradoxal que la Truite, sélectionnée en fonction de ses performances en pisciculture, puisse être considérée, sans la moindre interrogation, comme satisfaisante pour empoissonner les milieux sauvages ".

Compte tenu de ces remarques et observations, DONALDSON et al. (1957) pour la Truite cou coupé, Salmo clarkii, et FLICK et WEBSTER (1976) pour I'Omble de fontaine, ont testé des hybrides intra spécifiques entre des souches sauvages et domestiques. Dans les deux cas, après déversement en lac, à taille égale, les hybrides ont présenté un certain nombre d'avantages sur les souches domestiques (nombre de captures plus important, meilleure survie et, dans certaines occasions, meilleure croissance).

En France le recours à de tels hybrides a été préconisé par CUINAT (1971) qui pense que cette " solution de compromis " donne des " alevins de la génération F1, c'est-à-dire issus directement du croisement entre sujets sauvages et d'élevage. (qui) ne présentent guère plus de difficultés d'élevage que leurs congénères domestiques. Par contre, après élevage en conditions identiques, ils devraient présenter des caractères de rusticité supérieurs à ceux-ci ".

Dans le but de tester l'intérêt d'une telle pratique, nous avons comparé dans la présente expérimentation, menée avec la Truite commune Salmo trutta, les performances d'un hybride, obtenu à partir de mâles sauvages et de femelles domestiques et de la souche domestique : ces performances ont été mesurées dans un premier temps en élevage classique, en conditions identiques, et dans un second temps en ruisseau.

\section{MATÉRIELS ET MÉTHODES}

\section{- Prélèvements des gamètes et insémination artificielle.}

Les géniteurs d'origine domestique provenaient de la pisciculture interfédérale de la Gouarnais (Morbihan).

Les mâles sauvages étaient originaires du ruisseau d'Avion, affluent du Couesnon en Ille-et-Vilaine (figure n० 1 ).

Les pontes de deux groupes de femelles domestiques, A et B, ont été divisées en deux lots d'environ 20000 ovules qui ont été inséminés

- liun par un mélange de sperme de 10 mâles sauvages (S) qui avait été prélevé la veille puis transporté suivant le protocole décrit par MAISSE et PORCHER (1981),

- l'autre par un mélange de sperme de 10 mâles domestiques (P). L'insémination artificielle a été pratiquée suivant la technique utilisant le dilueur d'insémination " 532 "(BILLARD, 1977)

Quatre lots, (AS), (AP), (BS), et (BP) ont été ainsi constitués. L'incubation a eu lieu jusqu'au stade œillé à la Gouarnais. Les pourcentages d'embryonnement ont été calculés, 220 degrés-jours après fécondation, sur deux échantillons prélevés dans chacun des lots juste après le durcissement des œufs et incubés dans un circuit fermé thermorégulé $\left(10^{\circ} \mathrm{C}\right)$

- Etude des performances des différents lots pendant l'élevage en pisciculture.

Les œufs embryonnés ont été transférés à la pisciculture fédérale de Cardroc (IIle-et-Vilaine), dans des auges d'alevinages $(\mathrm{L}=2,2 \mathrm{~m} . \mathrm{l} .=0,6 \mathrm{~m}$., $\mathrm{h}$. $=0,17 \mathrm{~m}$., débit $15 \mathrm{l} / \mathrm{mn}$ ) où la croissance pondérale (pesée toutes les trois semaines) et la survie (relevé quotidien des mortalités) ont été contrôlées dans chaque lot jusqu'à trois mois après éclosion.

Pour chacune des périodes comprises entre deux pesées, le taux de croissance instantané $(G)$ de chacun des lots a été calculé selon RICKER (1979)

$$
G=\frac{\log W_{2}-\log W_{1}}{t_{2}-t_{1}}
$$




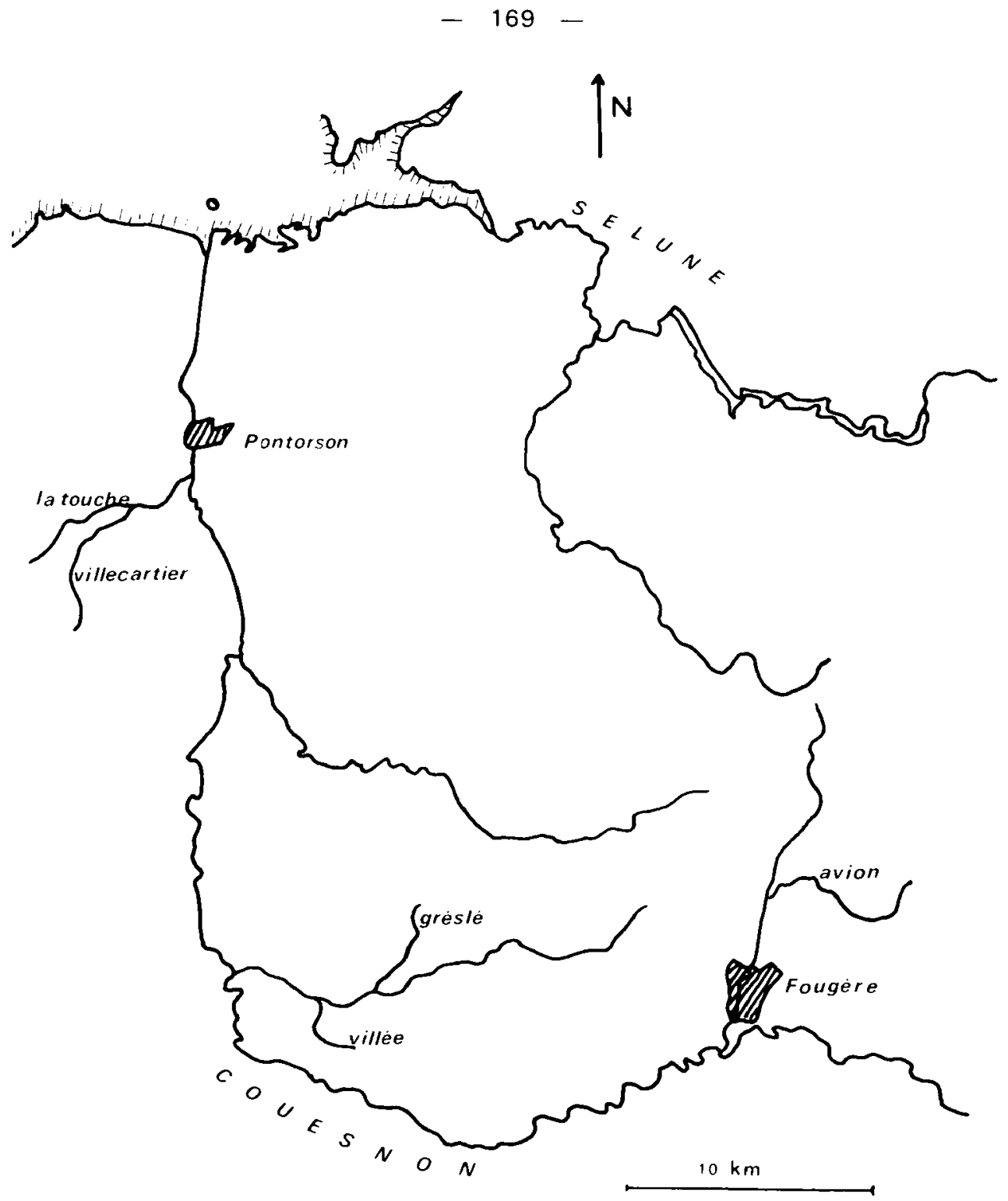

Figure 1: Situation des affluents du Couesnon où s'est déroulée l'étude.

où $W_{2}$ est le poids moyen (en g) du lot considéré à la date $t_{2}$ (en jours) et $W_{1}$ celui à la da te t1. Les fluctuations de la température de l'eau pendant cette période sont rapportées dans la figure $n^{\circ} 2$. Pendant les trois premières semaines d'alimentation, un aliment sec du commerce (I) et un aliment expérimental semi-humide (II) ont été distribués. Huit lots d'environ 10000 alevins ont ainsi été constitués :

- $A S_{1}, A P_{1}, B S_{1}, B P$

$-A S_{11}, A P_{11}, B S_{11}, B P_{11}$

La pesée réalisée au terme de la $3^{e}$ semaine d'alimentation, ayant mis en évidence une absence de croissance pour les lots nourris avec l'aliment II, nous avons décidé de distribuer l'aliment I aux huit lots.

A la fin du $1^{\text {er }}$ mois d'alimentation, l'effectif de chaque lot a été ramené à 5000 pour éviter une surcharge dans les lots à plus forte croissance.

Une costiose s'étant déclarée quand la température s'est établie au-dessus de $10^{\circ} \mathrm{C}$, nous avons procédé au traitement de tous les lots par des bains de formol (GERARD, 1981). 


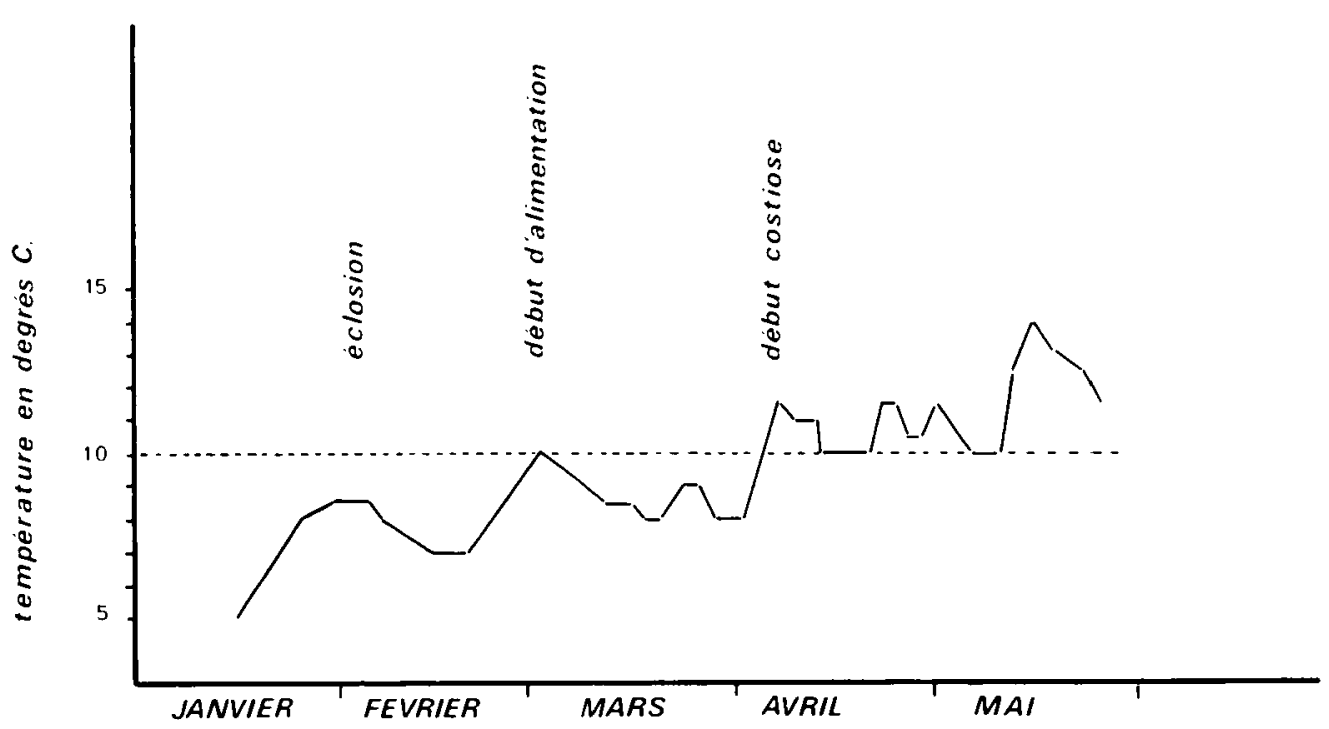

Figure 2: Température de l'eau d'élevage.

\section{produits en pisciculture.}

Quatre ruisseaux, affluents du Couesnon, ont été retenus pour cette étude (figure $\left.n^{\circ} 1\right)$. Sur chacun de ces ruisseaux, la première semaine de Juin, un secteur de $800 \mathrm{~m}$ a été ensemencé, uniformément, par un mélange de 800 juvéniles domestiques et de 800 juvéniles hybrides, (lots $A P_{11}$ et $A S_{\|}$sur le Greslé, BP, et $B S_{1}$ sur la Villée, $A P_{1}$ et $A S_{1}$ sur la Touche, $\mathrm{BP}_{11}$ et $\mathrm{BS}_{\|}$sur Villecartier) pris au hasard dans leur lot d'origine.

GREENE (1952), DONALDSON et al. (1957), VINCENT (1960) et FLICK et WEBSTER (1964 et 1976), utilisaient le marquage par ablation d'une nageoire pelvienne dans des études comparables à la nôtre. FLICK et WEBSTER (1976) précisant qu'ils n'ont pas observé les mortalités importantes rapportées par NICOLA et CORDONE (1973) à la suite de tels marquages, nous avons identifié les juvéniles domestiques par l'ablation de la nageoire pelvienne droite et les juvéniles hybrides par celle de la nageoire pelvienne gauche.

Après marquage, les poissons ont été mis en observation pendant 8 jours et une alimentation supplémentée en Vitamine $C(6 \mathrm{~g}$ par $100 \mathrm{~kg}$ de poids vif) a été donnée 3 jours de suite. Aucune mortalité notable n'a été observée pendant cette période.

A l'époque des déversements, sur les ruisseaux du Greslé et de la Villée, la taille des juvéniles autochtones, échantillonnés en amont des secteurs, ne différait pas significativement de celle des domestiques et était supérieure à celle des hybrides (STUDENT, $p=0,001)$.

Dans chacun des secteurs de déversement, des sous-secteurs de recapture ont été caractérisés (Tableau $n^{\circ} 1$ ). Ces sous-secteurs étaient distants des zones non alevinées de $200 \mathrm{~m}$ au minimum.

La dernière semaine de Septembre, ces secteurs ont été pêchés à l'électricité selon la méthode de DE LURY avec deux passages.

Une estimation des populations en place dans chaque sous-secteur a été calculée avec la formule de SEBER et LECREN :

$$
\mathrm{p}=\frac{\mathrm{c}_{1}{ }^{2}}{\mathrm{C}_{1}-\mathrm{C}_{2}}
$$




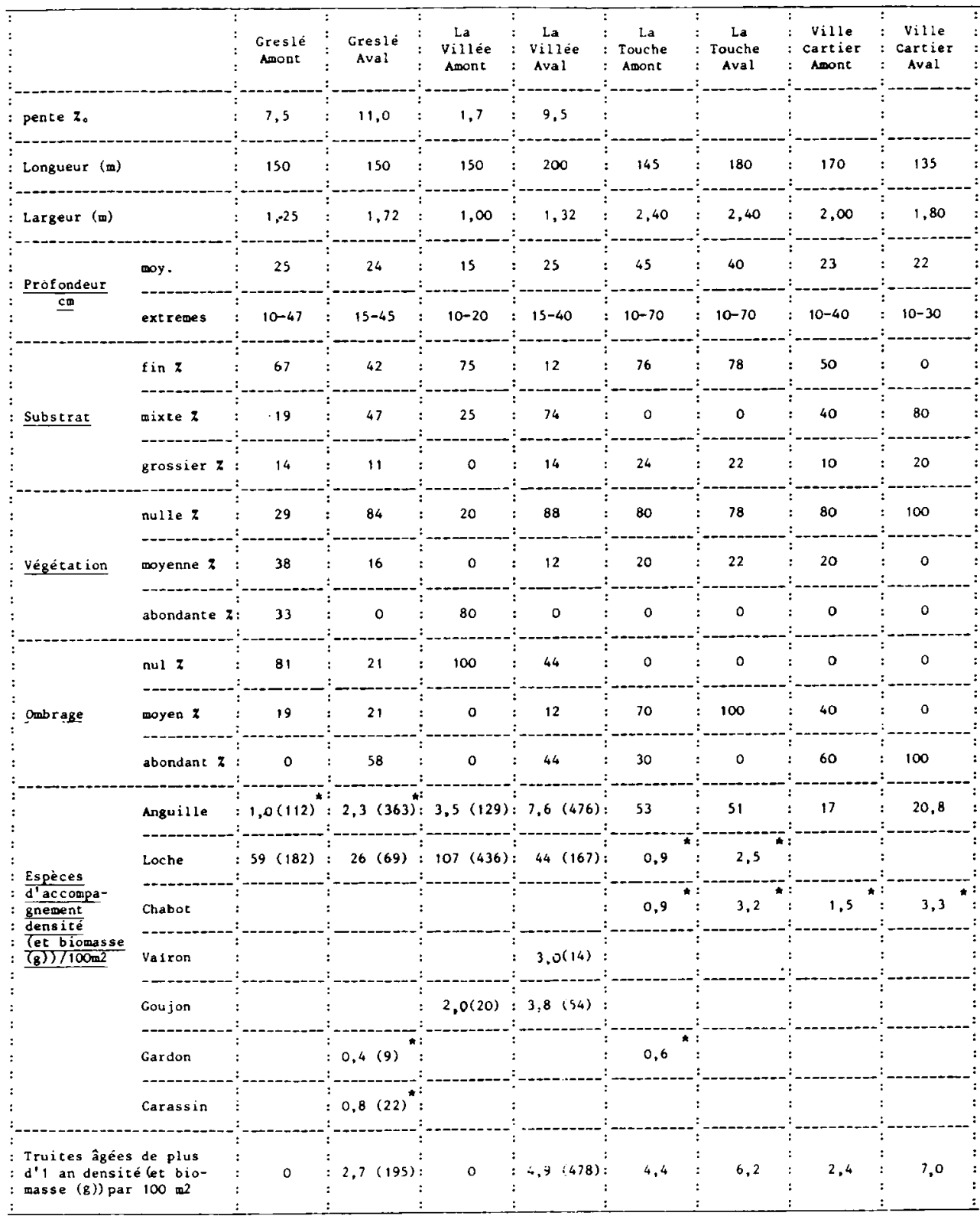

Tableau 1 : Caractéristiques des secteurs de recaptures: espèces d'accompagnement et Truites de plus d'un an présentes à la pêche électrique de Septembre.

\section{* Population pêchée.}

où $p$ est l'estimation du peuplement, $C_{1}$, l'effectif capturé à la première pêche et $C_{2}$, l'effectif capturé à la deuxième pêche.

Pour chaque sous-secteur la densité de chaque peuplement a été calculée pour $100 \mathrm{~m}^{2}$, et le taux d'implantation théorique des juvéniles de chaque lot a été calculé comme suit :

Taux d'implantation théorique : $\frac{\text { Densité estimée de juvéniles recapturés }}{\text { Densité théorique de juvéniles déversés. }} \times 100$


A la pêche de septembre, ont été considérés comme autochtones tous les juvéniles ayant les deux nageoires pelviennes de taille égale.

Dans l'ensemble des truites autochtones, la séparation entre les juvéniles $\mathrm{O}^{+}$an et les individus âgés de $1^{-}$an et plus a été établie d'après les histogrammes de fréquence des tailles (longueur à la fourche en $\mathrm{mm}$ )

Pour chacun des juvéniles capturés dans les ruisseaux du Greslé et de la Villée. le coefficient de condition (C) a été calculé à partir de la longueur à la fourche $(\mathrm{L}$, en $\mathrm{cm})$ et du poids $(P$, en $g$ ) avec la formule :

$$
C=\frac{100 P}{L^{3}}
$$

Enfin dans chacun des sous-secteurs et pour chaque lot, le taux de croissance instantanée $(G)$ a été calculé pour la période comprise entre le déversement et la recapture.

\section{- Tests statistiques :}

Les différences éventuelles entre les paramètres suivants ont été analysées par analyse de variance à plusieurs facteurs en modèle mixte :

- taux d'embryonnement $(2$ facteurs avec répétition, transformation angulaire des proportions),

- poids en fin de résorption ( 3 facteurs avec répétition)

- croissance en pisciculture ( 3 facteurs sans interaction).

- survie en pisciculture ( 3 facteurs sans interaction) à l'aide du taux quotidien de mortalité $(m)$

$$
m=1-\sqrt[t]{\frac{n t}{n o}}
$$

où no est le nombre initial et nt le nombre d'individus vivants au teme jour.

- croissance et coefficient de condition des juvéniles capturés en septembre ( 3 facteurs sans interaction).

La variabilité des poids dans chacun des lots a été étudiée à l'aide du test de WILCOXON, en fin de résorption, puis du test de BARTLETT. Entre les deux sous-secteurs du même ruisseau, l'importance relative des peuplements estimés des juvéniles hybrides, d'une part, et domestiques d'autre part, a été analysée à l'aide du chi-carré.

\section{RESULTATS :}

- Taux d'embryonnement : (Tableau n²)

Aucune différence significative ne peut être décelée suivant l'origine du sperme.

- Croissance en élevage : (figure $n^{\circ} 3$, Tableaux $n^{\circ} 3$ et 4)

- Aucune différence significative n'a été décelée entre les deux groupes de femelles utilisées.

- Un effet "mâle " en faveur de l'origine domestique est observé dès la fin de la résorption vésiculaire et reste constamment significatif en ce qui concerne les poids moyens des différênts lots.

- Cependant, l'analyse des taux de croissance instantanée indique que l'effet " mâle " est significatif jusqu'au 12 mai, date à partir de laquelle aucune différence significative n'est décelée pour ce paramètre.

- L'utilisation de l'aliment Il se traduit le 3 mars par un effet défavorable sur la croissance. Cet effet s'estompe à partir du 22 avril après la distribution, à tous les lots, de l'aliment I.

- Par contre, la variabilité plus grande des poids des alevins dans les lots nourris avec l'aliment II se maintient jusqu'au 12 mai, pour disparaitre le 25 mai. 


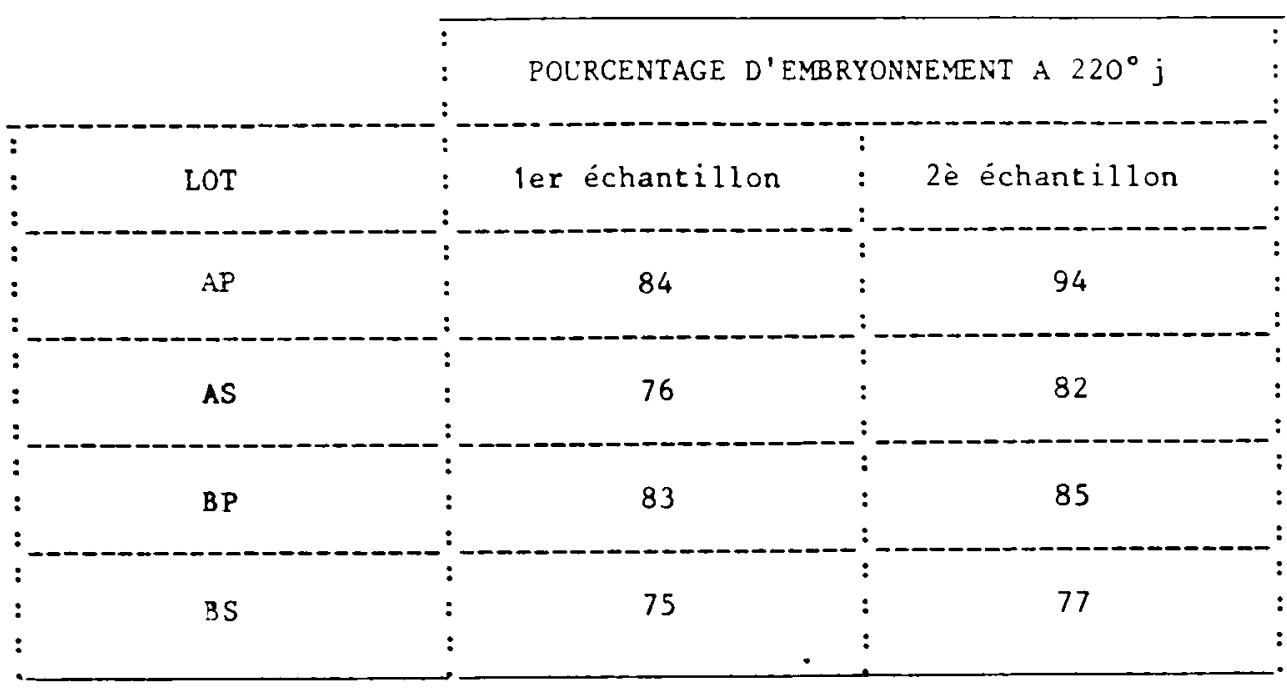

Tableau 2: Pourcentage d'embryonnement 220 degrés-jours après fécondation dans les divers lots.

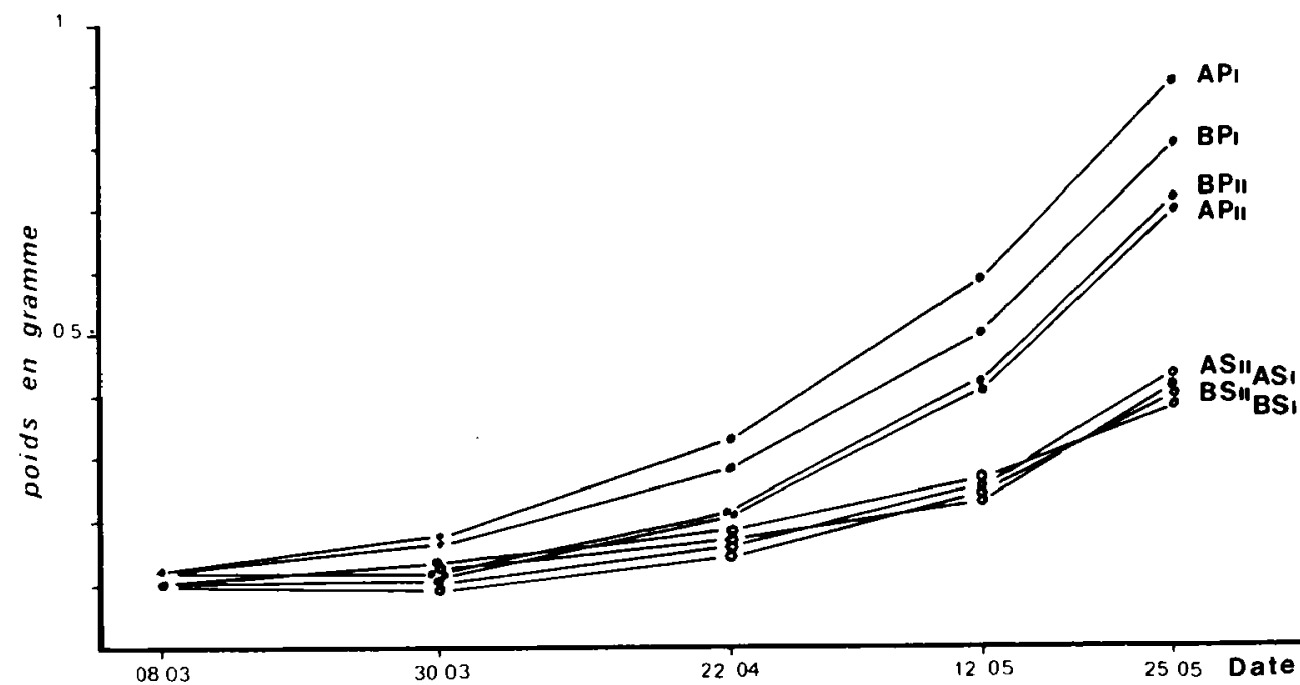

Figure 3: Croissance moyenne des différents lots pendant la phase d'élevage :

- lots domestiques $A P_{1}, A P_{\mid 1}, B P_{1}, B P_{\mid 1}$

- lots hybrides $A S_{1}, A S_{\|}, B S_{1}, B S_{\|}$.

\section{- Mortalité en élevage (figure n० 4, Tableau n 5)}

Aucune différence notable de mortalité n'est apparue, jusqu'à l'apparition de l'épidémie de Costiose qui s'est manifestée en deux phases :

- une phase aiguë du 6 au 14 avril

- une phase chronique à partir du premier traitement au formol le 14 avril.

Durant la phase aiguë, les mortalités sont significativement plus importantes dans les lots hybrides que dans les autres. Cet effet mâle est plus évident encore dans la phase chronique. 


\begin{tabular}{|c|c|c|c|c|c|c|c|c|c|c|c|}
\hline$: \quad$ Date & $\begin{array}{l}: \\
\vdots \\
:-\end{array}$ & $\begin{array}{l}\text { mars } \\
\text { résorp }\end{array}$ & 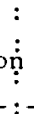 & 30 mars & $\begin{array}{l}: \\
: \\
:\end{array}$ & 22 avril & $\begin{array}{l}: \\
\vdots \\
\vdots\end{array}$ & $12 \mathrm{mai}$ & $\begin{array}{l}: \\
:\end{array}$ & 25 mai & \\
\hline $\begin{array}{l}: \text { Effet } \\
: \text { "femelle" } \\
:\end{array}$ & $\begin{array}{l}\vdots \\
\vdots \\
:\end{array}$ & N.S. & $:$ & N.S. & $\begin{array}{l}: \\
\vdots \\
:\end{array}$ & N.S. & $\begin{array}{l}: \\
: \\
:\end{array}$ & N.S. & $\begin{array}{l}: \\
:\end{array}$ & N.S. & \\
\hline $\begin{array}{l}: \text { Effet } \\
: \quad \text { "Iâle" }\end{array}$ & $\begin{array}{l}: \\
:\end{array}$ & s. & $\begin{array}{l}: \\
: \\
:\end{array}$ & S. & $\begin{array}{l}: \\
:\end{array}$ & S. & $\begin{array}{l}: \\
:\end{array}$ & S. & $:$ & S. & \\
\hline $\begin{array}{l}: \text { Effet } \\
: \text { "aliment" } \\
:\end{array}$ & $:$ & N.S. & $\begin{array}{l}: \\
:\end{array}$ & S. & $\begin{array}{l}: \\
: \\
:\end{array}$ & N.S. & $\begin{array}{l}: \\
:\end{array}$ & N.S. & $\begin{array}{l}: \\
:\end{array}$ & N.S. & \\
\hline $\begin{array}{l}: V a r i a b i l i t \\
:\end{array}$ & $:$ & N.S. & $\begin{array}{l}: \\
\vdots \\
:\end{array}$ & S. & $\begin{array}{l}: \\
\vdots \\
\vdots\end{array}$ & S. & $\begin{array}{l}\vdots \\
\vdots \\
:\end{array}$ & s. & $\begin{array}{l}: \\
:\end{array}$ & N.S. & \\
\hline
\end{tabular}

Tableau 3 : Analyse des différences observées dans les poids moyens des différents lots (figure $n^{\circ} 3$ ) (La ligne " Variabilité " traduit la signification de différences observées dans la dispersion des poids suivant le régime alimentaire).

N.S. = non significatif $(p=0,05)$

S. = significatif $(p=0,05)$

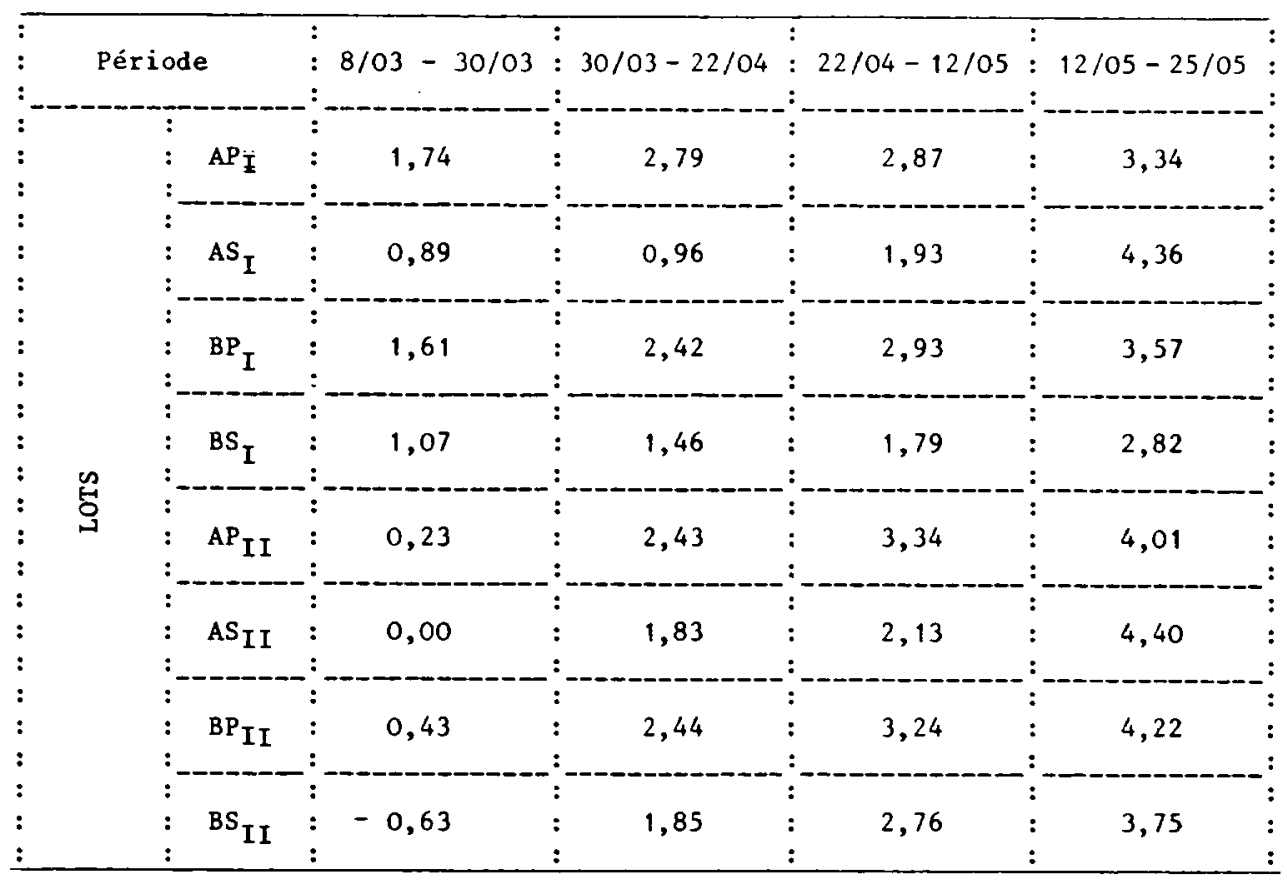

Tableau 4 : Evolution du taux de croissance instantanée de chacun des lots en fonction de la période pendant l'élevage.

Dans cette dernière phase, les traitements au formol arrêtent pratiquement toute mortalité dans les lots domestiques et non chez les hybrides où la présence de costia est toujours détectée sur les individus mourants.

Aucun effet " femelle "ou "aliment "n'est apparu dans l'analyse des mortalités. 


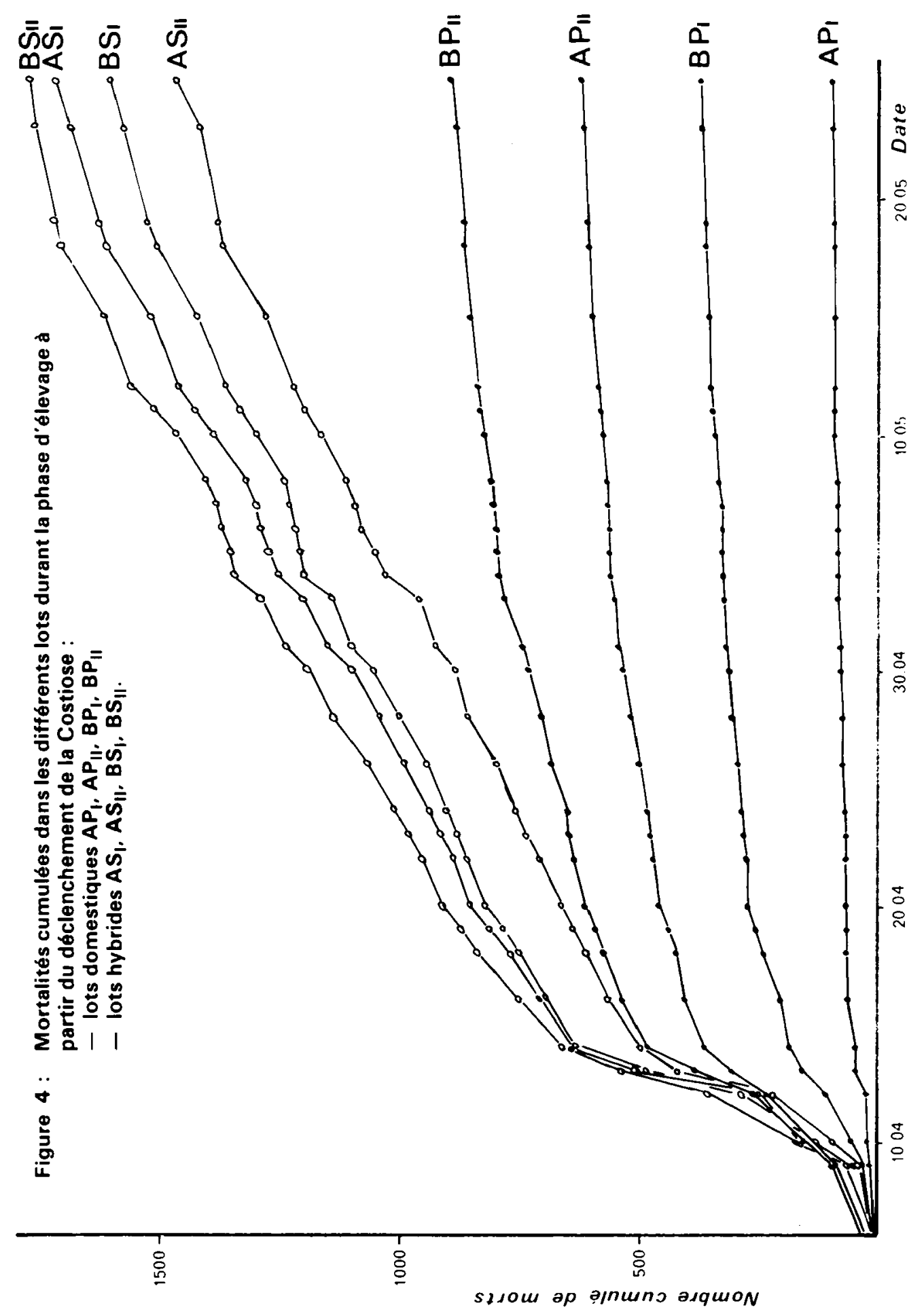




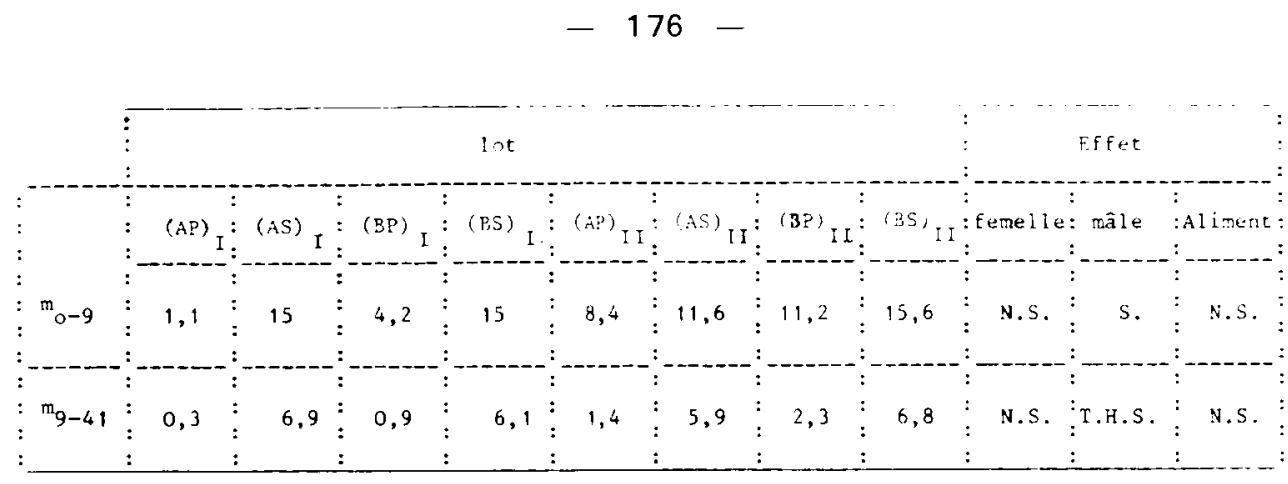

Tableau 5: Analyse des mortalités (figure $n^{\circ} 2$ ):

$\mathbf{m}_{0.9}=$ taux quotidien de mortalité pendant la phase aiguë $(\% 0)$

$m_{9.41}=$ taux quotidien de mortalité pendant la phase chronique $(\% 0)$

N.S. = non significatif $(p=0,05)$

S. = significatif $(p=0,05)$

T.H.S. $=$ significatif $(p=0,001)$.

\section{- Implantation en ruisseau :}

Quatre mois après les déversements en ruisseau, l'analyse des peuplements suivant les origines (Tableau $n^{\circ} 6$ ) montre que dans les ruisseaux où l'implantation a été la meilleure, les sous-secteurs amont semblent plus favorables aux hybrides (non significatif à $p=0,05$ pour le ruisseau du Greslé, significatif à $p=0,01$ pour le ruisseau de la Villée, $X^{2}$ calculés sur les effectifs de chacun des sous-secteurs) alors que les densités de juvéniles autochtones varient peu.

\section{- Croissance en ruisseau :}

L'analyse des caractéristiques des juvéniles recapturés en septembre sur les ruisseaux du Greslé et de la Villée indique : (Tableau $n^{\circ}{ }^{7}$ )

- un effet " ruisseau ", en faveur de la Villée, significatif à $1 \%$ sur la taille, à $1 \%$ sur le poids et à $1 \%$ sur le gain de poids.

- un effet " amont-aval "en faveur des sous-secteurs amont significatif à $1 \%$ sur la taille et non significatif à $5 \%$ sur le poids,

- un effet " mâle ", en faveur de l'origine domestique significatif à $1 \%$ sur la taille finale, à $1 \%$ sur le poids final. Cependant, dans chaque sous-secteur, les hybrides et les domestiques ont un taux de croissance instantanée identique ;

- aucun effet significatif (à $5 \%$ ) n'apparaît dans l'étude des coefficients de condition.

\section{DISCUSSION - CONCLUSION}

\section{Phase d'élevage}

Les bons résultats de l'insémination artificielle avec du sperme de mâles sauvages, après transport, confirment ceux obtenus par MAISSE et PORCHER (1981) dans les mêmes conditions.

D'une manière générale, l'élevage de l'hybride s'est révélé difficile avec une croissance faible et une mortalité importante. Dès la fin de la résorption vésiculaire, le poids des hybrides est significativement inférieur à celui des domestiques. Ceci est sans doute à relier à une activité spontanée plus, importante des hybrides lors d'une résorption effectuée sur des clayettes de pisciculture à fond lisse. De telles différences de comportement ont été rapportées notamment par GREENE (1952) et VINCENT (1960) entre des Ombles de fontaine sauvages et domestiques. Suite aux observations de MARR (1963) relatives à l'influence de la structure des incubateurs sur le comportement des alevins de salmonidés, un certain nombre d'auteurs, dont LEEON (1975), LEEON et BONNEY (1979). GAIGNON et PROUZET (1982), ont montré que les alevins ayant résorbé leur vésicule sur un substrat irrégulier sont plus gros en fin de résorption que ceux issus d'incubateurs à fond lisse. LÉON et BONNEY (1979) ont aussi montré, chez le Saumon atlantique, Salmo 


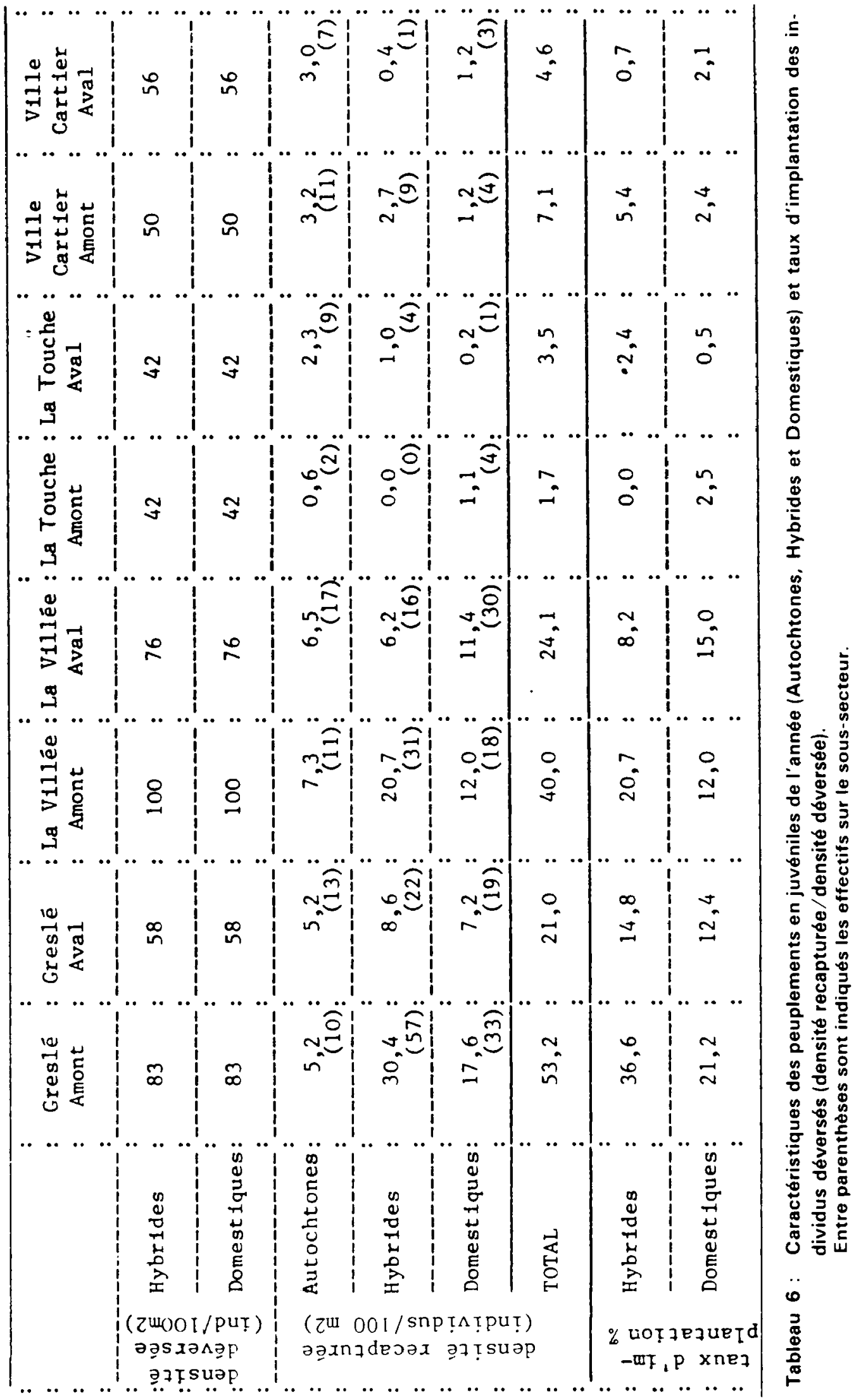




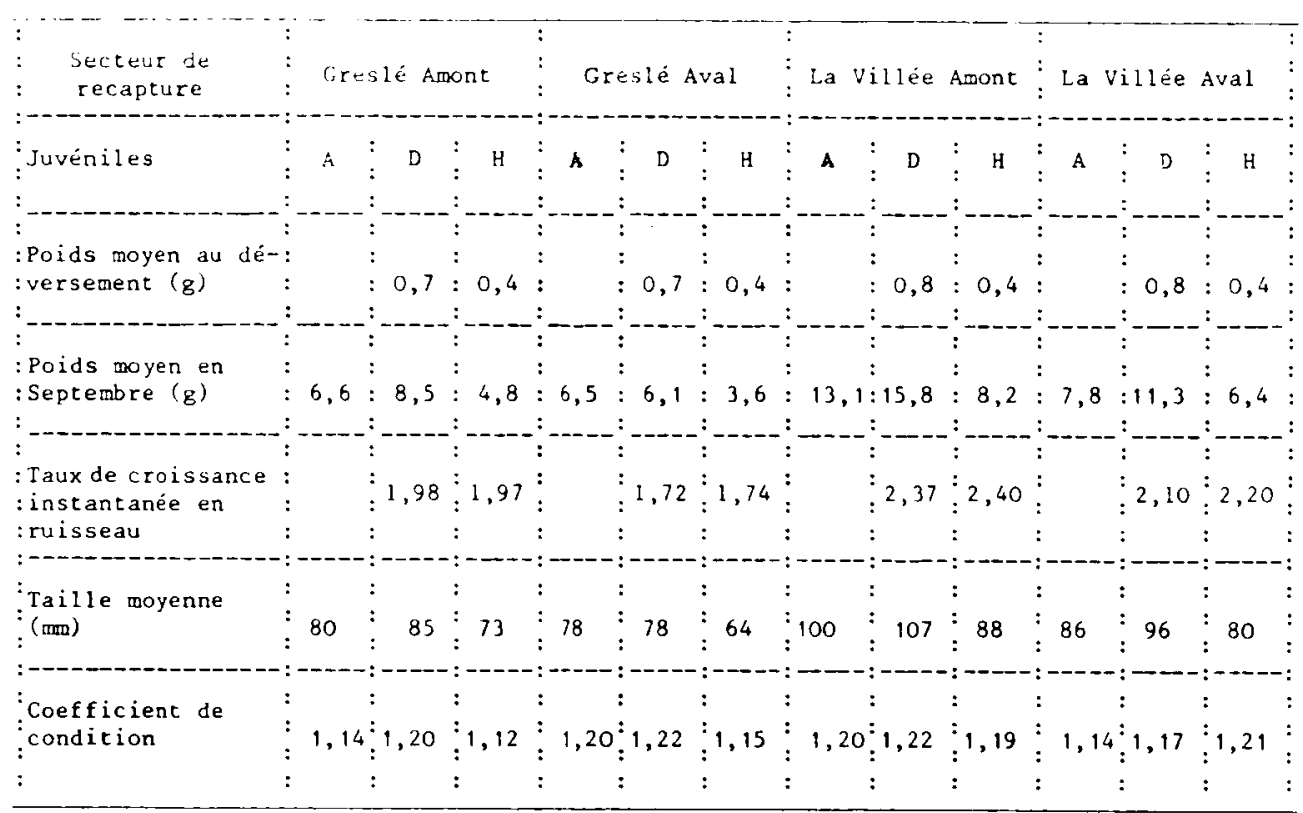

Tableau 7: Caractéristiques de taille et de poids des juvéniles de Truite capturés sur les ruisseaux du Greslé et de la Villée.

$A=$ juvéniles autochtones : $D=$ juvéniles domestiques : $H=$ juvéniles hybrides.

salar, que cet avantage de poids se conserve au moins jusqu'à 45 jours après le début de l'alimentation. II est donc possible que les moins bonnes performances de croissance enregistrées chez les hybrides proviennent des conditions de résorption et que les résultats soient différents en utilisant d'autres substrats.

II apparaît cependant que les hybrides survivant le 12 mai s'adaptent aux conditions d'élevage puisqu'ils présentent à partir de cette date un taux de croissance instantanée comparable à celui des domestiques.

Enfin, la plus grande sensibilité des hybrides à la Costiose ne nous semble pas devoir être attribuée directement à des caractères génétiques particuliers, mais plutôt à la grande différence de taille en faveur des domestiques qui étaient deux fois plus gros à cette époque.

Par ailleurs, les mortalités chroniques enregistrées chez les hybrides sont à rapprocher de celles observées par LEON et BONNEY (1979) sur des Saumons atlantiques ayant résorbé leur vésicule sur fond lisse.

En conclusion, nos résultats confirment ceux obtenus par FLICK et WEBSTER (1976) dans l'élevage d'un hybride semblable d'Omble de fontaine (mauvaise croissance, mortalité importante) et nous pensons que les performances de tels hybrides peuvent être grandement améliorées par l'utilisation des techniques propres à l'élevage du Saumon atlantique, telles celles décrites par THORPE (1981) : résorption sur fond cannelé avec luminosité réduite par exemple.

\section{Phase en ruisseau}

L'importance relative des juvéniles des deux origines apparaît liée aux caractéristiques du secteur. Ainsi, le sous-secteur " La Villée amont " qui a une pente faible, une profondeur faible, un substrat fin, une végétation abondante et un ombrage nul, est favorable aux hybrides alors que le sous-secteur " La Villée aval "qui a une pente plus forte, mais une hauteur d'eau plus importante avec de nombreux profonds, un substrat en majorité mixte, une végétation quasiment nulle et un ombrage important, est favorable aux domestiques. 
Cette observation est à rapprocher de celle de VINCENT (1960) qui constate une meilleure implantation de juvéniles d'O mble de fontaine d'origine sauvage comparée à celle de juvéniles domestiques, dans les secteurs d'un ruisseau caractérisés par un courant rapide et une absence de profonds, les proportions sauvages-domestiques s'équilibrant ou s'inversant dans les autres secteurs. VINCENT explique cette constatation par une flotabilité différente des domestiques qui ont tendance à nager en surface et des sauvages qui nagent près du fond, observation confirmée avec la Truite commune par SOSIAK (1982) qui avance une explication alimentaire (teneur en lipides de l'aliment), voire génétique. Une autre explication de ces différences de répartition suivant les sous-secteurs peut être donnée à partir de l'étude de la densité en truites âgées de plus d'un an, que l'on trouve surtout dans les profonds. En effet, il existe une corrélation négative $(84 \%)$ entre la densité de ces classes d'àge et celle des juvéniles hybrides. (71\% pour les domestiques et $56 \%$ pour les résidents).

On peut donc penser que la faible taille des hybrides déversés les a rendus plus vulnérables que les domestiques à la prédation par les truites àgées de plus d'un an. Cette prédation pourrait agir en synergie avec les autres caractétistiques physiques et hydrauliques des secteurs.

Les caractéristiques des juvéniles recapturés en septembre indiquent que les juvéniles domestiques gardent leur avantage en taille, comme l'avait observé VINCENT (1960) sur des Ombles de fontaine d'origines sauvage et domestique déversés en ruisseau et en lac.

Par contre FLICK et WEBSTER (1976) observent parmi les individus recapturés à la ligne une meilleure croissance des hybrides, leur taille de déversement étant assez proche de celle des domestiques $(9,1 \mathrm{~cm}$ et $9,4 \mathrm{~cm})$. Dans notre expérimentation, le fait que les hybrides et les domestiques présentent, dans chacun des sous-secteurs, des taux de croissance instantanée identiques, tend à montrer que ces individus subissent l'influence discriminante des conditions d'élevage préalable uniquement par leur décalage de taille au déversement, et celle du milieu " ruisseau " uniquement par le taux d'implantation en fonction des caractéristiques physiques et hydrauliques.

Cette hypothèse est renforcée par l'absence de différence significative entre les coefficients de condition des individus suivant leur origine. Ces différentes observations nous amènent donc à distinguer nettement, pour juger de l'intérêt de tels hybrides, trois stratégies de déversement correspondant à des objectifs différents.

Une stratégie " de recapture " dans laquelle les animaux déversés sont ceux destinés à être repris. Dans ce cas, l'utilisation de juvéniles " domestiques " semble assurer, comparativement aux hybrides, de meilleures performances lors de la phase d'élevage tout en permettant une implantation correcte et une bonne croissance dans le milieu sauvage. Ces animaux doivent cependant être capturés dans leur quasi totalité (voire stérilisés) si l'on veut éviter les risques de pollution génétique des populations autochtones. REISENBICHLER et McINTYRE (1977) ont en effet montré chez la Truite "tête d'acier" (Salmo gairdneri) que lorsque des géniteurs d'origine pisciculture se croisaient avec des géniteurs sauvages, on pouvait aboutir à une diminution du nombre de smolts produits. Dans ce premier cas, l'utilisation d'hybrides ne semble donc pas se justifier, contrairement aux résultats déjà cités de DONALDSON et al. (1957) et FLICK et WEBSTER (1976). L'obtention d'une " vigueur hybride " par croisement entre souches de Salmonidés ne peut donc être considérée comme systématique, comme le montrent également les résultats négatifs de plusieurs auteurs ayant testé de tels croisements en élevage ou dans le milieu naturel (KLUPP, 1979 ; BAILEY et SAUNDERS, 1979 ; BAILEY et al., 1980 ; GJERDE, 1981); comme il est d'autre part classiquement admis qu'une condition nécessaire à l'obtention d'une telle vigueur est l'utilisation de souches génétiquement différenciées, la remarque concernant la nécessité d'une élimination quasi totale des individus déversés s'applique en outre tout aussi bien à de tels hybrides qu'à des individus de souches domestiques.

Une stratégie " de soutien des populations " dans laquelle les alevins déversés doivent venir renforcer un peuplement en place considéré comme insuffisant. Dans ce cas on souhaite donc déverser des individus génétique ment proches des animaux autochtones. Si des alevins " hybrides "apparaissent répondre partiellement à cette exigence, en réalisant un compromis entre facilité de production et utilisation de gènes " sauvages ", il apparaît souhaitable cependant de n'utiliser comme souche femelle que des animaux eux-mêmes génétiquement très proches de la population sauvage considérée (la souche peut être constituée à partir de gamètes prélevés sur des géniteurs sauvages. 
et entretenue par un apport fréquent de gènes "sauvages ") Dans le cas contraire, le risque d'apparition d'individus génétiquement inadaptés dans la descendance des hybrides apparaît notable compte tenu du manque de connaissances actuelles sur la différenciation génétique des différentes populations de Truite commune (GUYOMARD et KRIEG, 1983). Indiquons notamment que des populations de Truite commune se comportant comme des espèces biologiques différentes ont été identifiées en Suède (ALLENDORF et al, 1976).

Une stratégie" de peuplement "d'un milieu d'accueil vierge ou accidentellement dépeuplé. Dans ce cas les alevins hybrides peuvent représenter un vecteur privilégié pour introduire dans le milieu une large variabilité génétique ultérieurement soumise à la sélection. On peut en effet envisager la collecte d'échantillons de sperme dans de nombreuses souches sauvages et la réalisation de populations " synthétiques "regrou pant les différents croisements possibles. Dans ce cas, un déversement au stade d'œufs embryonnés est peut-ètre d'ailleurs à envisager pour permettre une action plus précoce de la sélection naturelle (SAUNDERS et BAILEY, 1980).

Signalons que, dans le cas des deux premiers types de déversement, le problème de l'efficacité réelle de telles pratiques reste posé car, dans notre expérimentation, nous ne sommes pas en mesure de préciser si ies juvéniles déversés, qui se sont implantés, sont venus en complément des juvéniles autochtones ou s'ils ont pris la place d'un certain nombre d'entre eux.

Enfin, il faut préciser que nos résultats ne nous permettent pas de préjuger de ceux qui seraient obtenus avec des individus déversés à des stades plus précoces

\section{REMERCIEMENTS}

Que les Fédérations Départementales des Associations Agréées de Pêche et de Pisciculture d'llle-et-Vilaine et du Morbihan soient ici remerciées pour l'aide qu'elles nous ont apportée tout au long de cette étude

\section{BIBLIOGRAPHIE}

ALLENDORF F.W., RYMAN N., STENNEK A., STAHL G., 1976. Genetic variation in scandinavian population of Brown trout (Salmo trutta L.) : evidence of genetically distinct sympatric population. Hereditas, 83, 73-82

BAILEY J.K., SAUNDERS R.L., 1979. Preliminary report on releases and returns of two year-classes of Atlantic salmon smolts from various pure strains and strain crosses. North American Salmon Research Center Report n० 5, 7 p

BAILEY J.K., BUZETA M.I., SAUNDERS R.L., 1980. Returns of three year-classes of sea ranched Atlantic salmon of various river strains and their hybrids. North American Salmon Research Center Report n³, 10 p.

BILLARD R., 1977. Utilisation d'un système tris-glycocolle pour tamponner le dilueur d'insémination pour la truite. Bull. Fr. Piscic., 264, 102-112.

CUINAT R., 1971. Ecologie et repeuplement des cours d'eau à truites. Bull. Fr. Piscic., $240,242,243,87 \mathrm{p}$

DONALDSON L.R., HANSLER D.D., BUCKRIDGE T.N., 1957. Interracial hybridization of Cutthroat trout, Salmo clarkii, and its use in fisheries management. Trans. Am. Fish. Soc., 86, 350-360

FLICK W.A., WEBSTER D.A., 1976. Production of wild, domestic and interstrain hybrids of Brook trout (Salvelinus fontinalis) in natural ponds. J. Fish. Res. Board Can.. 33, 1525-1539

FLICK W.A., WEBSTER D.A., 1976. Production of wild, domestic and luterstrain hybrids of Brook trout (Salvelinus fontinalis) in natural ponds. J. Fish. Res. Board Can., 33, $1525-1539$.

GAIGNON J.L., PROUZET P., 1982. Elevage du Saumon atlantique ( $S$. salar) en écloserie : essai d'utilisation de substrats durant la résorption vitelline. Bull. Fr. Piscic., $287,1-5$.

GERARD J.P., 1981. Le formol en thérapeutique piscicole. Bull. Fr. Piscic., 280, 111-112. 
GJERDE B., 1981. Growth rate and mortality of crosses between strains of Atlantic salmon. 32nd Annual Meeting of the European Association for animal production. ZAGREB, Yougoslavie.

GREENE C.W., 1952. Results from stocking Brook trout of wild and hatchery strains at still water pond. Trans. Am. Fish. Soc., 81, 43-52.

GREENE C.W., 1964. A comparison of stamina of Brook trout from wild and domestic parents. Trans. Am. Fish. Soc., 93, (1), 96-100.

GUYOMARD R., KRIEG F., 1983. Electrophoretic variation in six populations of Brown trout. (Salmo trutta L.). Can. J. of genetics and cytology, (in press).

KLUPP R., 1979. Genetic variance for growth in Rainbow trout (Salmo gairdneri L.). Aquaculture, 18, 123-134

LEON K.A., 1975. Improved growth and survival of juvenile Atlantic salmon (Salmo salar) hatched in drums packed with a labyrinthine plastic substrate. Prog. Fish Cult., 37. (3), 158-163.

LEON K.A., BONNEY W.A., 1979. Atlantic salmon embryos and fry : effects of various incubation and rearing methods on hatchery survival and growth. Prog. Fish Cu/t.. 41. (1), 20-25.

MAISSE G., PORCHER J.P., 1981. Une méthode de production de Truites fario destinées aux repeuplements de restauration. Bull. Fr. Piscic., 280, 134-144.

MARR D.H.A., 1963. The influence of surface contour on the behaviour of trout alevins, S. Trutta L.. Animal behaviour, 11, 412.

NICOLA S.J., CORDONE A.J., 1973. Effects of fin removal on survival and growth of Rainbow trout (Salmo gairdner/) in a natural environment. Trans. Am. Fish. Soc., 102, (4). 753-758.

REISENBICHLER R.R., McINTYRE J.D., 1977. Genetic differences in growth and survival of juvenile hatchery and wild steelhead trout. Salmo gairdneri. J. Fish. Res. Board Can., 34, 123-128.

RICKER W.E., 1979. Growth rates and model, 677-743 in HOAR W.S., RANDALL D.J., BRETT J.R. Fish Physiology, Volume VIII, Bioenergetics and growth, Academic Press, New-York, San Francisco, London.

SOSIAK A.J., 1982. Buoyancy comparisons between juvenile Atlantic salmon and Brown trout of wild and hatchery origin. Trans. Am. Fish. Soc., 111, 307-311

THORPE J.E., 1981. Rearing salmonids in freshwater, 325-344, in HAWKINS A.D., Aquarium Systems, Academic Press, London.

VINCENT R.E., 1960. Some influence of domestication upon three stocks of Brook trout (Salvelinus fontinalis Mitchill). Trans. Am.Fish. Soc., 89, 35-52. 